\begin{tabular}{|c|l|}
\hline Title & Clinical characteristics of pleomorphic carcinoma of the lung \\
\hline Author(s) & $\begin{array}{l}\text { Ito, Kenichiro; Oizumi, Satoshi; Fukumoto, Shinichi; Harada, Masao; Ishida, Takashi; Fujita, Yuka; Harada, Toshiy uki; } \\
\text { Kojima, Tetsuya; Y okouchi, Hiroshi; Nishimura, Masaharu; Hokkaido Lung Cancer Clinical Study Group }\end{array}$ \\
\hline Citation & $\begin{array}{l}\text { Lung Cancer, 68(2), 204.210 } \\
\text { https://doi.org/40.1016/.lungcan.2009.06.002 }\end{array}$ \\
\hline Issue Date & 2010-05 \\
\hline Doc URL & http://hdl.handle.net/2115/43068 \\
\hline Type & article (author version) \\
\hline File Information & LC682_204.210.pdf \\
\hline
\end{tabular}

Instructions for use 


\section{Clinical Characteristics of Pleomorphic Carcinoma of the Lung}

Kenichiro Ito ${ }^{a}$, Satoshi Oizumi ${ }^{a}$, Shinichi Fukumoto ${ }^{b}$, Masao Harada ${ }^{b}$, Takashi Ishida ${ }^{c}$, Yuka Fujita ${ }^{d}$, Toshiyuki Harada $^{\mathrm{e}}$, Tetsuya Kojima ${ }^{\mathrm{f}}$, Hiroshi Yokouchi ${ }^{\mathrm{g}}$, Masaharu Nishimura ${ }^{a}$, Hokkaido Lung Cancer Clinical Study Group

${ }^{a}$ First Department of Medicine, Hokkaido University School of Medicine, Sapporo, Japan

${ }^{\mathrm{b}}$ Department of Pulmonary Diseases, National Hospital Organization Hokkaido Cancer Center, Sapporo, Japan

${ }^{\mathrm{c}}$ Department of Pulmonary Medicine, Fukushima Medical University School of Medicine, Fukushima, Japan

${ }^{d}$ Department of Respiratory Medicine, National Hospital Organization Dohoku National Hospital, Asahikawa, Japan

${ }^{\text {e }}$ Section of Respiratory Diseases, Department of Internal Medicine, Hokkaido Social Insurance Hospital, Sapporo, Japan

${ }^{f}$ Department of Medical Oncology, KKR Sapporo Medical Center, Sapporo, Japan

${ }^{9}$ Department of Medicine, Hokkaido Chuo Rosai Hospital, Iwamizawa, Japan 


\section{Correspondence to:}

Satoshi Oizumi, First Department of Medicine, Hokkaido University School of Medicine, North 15, West 7, Kita-ku, Sapporo 060-8638, Japan. Phone: +81-11-706-5911; Fax: +81-11-706-7899; E-mail: soizumi@med.hokudai.ac.jp

\section{ABSTRACT}

Background: Pleomorphic carcinoma of the lung is a malignant epithelial tumor that contains carcinomatous and sarcomatoid components. Due to its rarity, few studies have been reported, and its clinical and pathological characteristics remain unclear.

Method: We retrospectively investigated 22 cases of pleomorphic carcinoma of the lung.

Results: Fifteen cases were diagnosed by surgical resection, four by autopsy, and three by transbronchial biopsy. Nineteen patients were male and three were female, and their mean age at diagnosis was 68.3 years $( \pm 10.1)$. Eighteen were current- or ex-smokers with substantial smoking histories (mean 46.4 pack-years). Sixteen patients had symptoms: hemoptysis and cough were commonly seen. Chest computed tomography (CT) findings revealed that the tumors were quite large (mean diameter $45.3 \pm 21.9 \mathrm{~mm}$; range 14-110 mm), and 21 tumors were peripherally located. Positron emission tomography with 18-fluorodeoxy-glucose (FDG-PET) was performed in 12 patients, and the Standardized Uptake Value (SUV) tended to be high (9.44 \pm 4.98$)$. In the 15 patients who underwent surgical resection, recurrence was common; systemic metastases were also frequently found. Patients who had received surgical 
treatment with proper follow-up care survived longer than those who did not undergo surgery. Responses to chemotherapy were generally poor, although one patient exhibited partial response to gefitinib.

Conclusions: Pulmonary pleomorphic carcinoma has strong malignant potential with frequent distant metastases, as has already been reported. However, this study demonstrated that surgical treatment and appropriate follow-up therapy might result in better prognoses.

Keywords: pleomorphic carcinoma; FDG-PET; surgery; chemotherapy; gefitinib; prognosis 


\section{Introduction}

According to the World Health Organization classification of lung tumors, pleomorphic carcinoma of the lung is one of five subgroups of sarcomatoid carcinoma [1], which itself is defined as a group of poorly differentiated tumors characterized pathologically by a combination of epithelial and mesenchymal elements. Pleomorphic carcinoma is histologically defined as either non-small cell carcinoma combined with neoplastic spindle and/or giant cell or a carcinoma that consists of only spindle cell and giant cell. At least $10 \%$ of the neoplasm should be pleomorphic.

If the tumor consists of only spindle cells or only giant cells, it is defined as a spindle cell or giant cell carcinoma, which are other subgroups of sarcomatoid carcinoma. Carcinosarcoma, which contains carcinoma and sarcoma, is another subgroup. Sarcoma components differentiate to osteosarcoma, chondrosarcoma, and rhabdomyosarcoma. Bone, cartilage, muscle, fat, and neuron are sometimes detected pathologically in tumors. Pulmonary blastoma is a very rare type of sarcomatoid carcinoma characterized as biphasic tumor containing a primitive epithelial component resembling well-differentiated fetal-type adenocarcinoma and a primary mesenchymal stroma.

Since its diagnostic criteria were confirmed, pulmonary pleomorphic carcinoma has been diagnosed more frequently. It is essential to understand its clinical behavior for effective management of patients with this disease. However, as pulmonary pleomorphic carcinoma is rare (only $0.1-0.4 \%$ of all malignant tumors of the lung) [2-4], its clinical and pathological characteristics 
are not well known. We have retrospectively investigated 22 patients with pulmonary pleomorphic carcinoma diagnosed by surgical resection, autopsy, or transbronchial biopsy (TBB). Radiological findings, results of positron emission tomography with 18-fluorodeoxy-glucose (FDG-PET), treatment, and clinical course are described in this report.

\section{Material and methods}

\subsection{Patients}

We retrospectively analyzed 22 pulmonary pleomorphic carcinomas from a total of 2447 primary lung cancers (including 1022 cases of resected lung cancer) that we experienced between January 2005 and December 2008 at seven institutes (Hokkaido University Hospital, National Hospital Organization Hokkaido Cancer Center, Fukushima Medical University School of Medicine, National Hospital Organization Dohoku National Hospital, Hokkaido Social Insurance Hospital, Hokkaido Chuo Rosai Hospital, and KKR Sapporo Medical Center). Pathological diagnosis was made by surgical resection, autopsy, or TBB. The incidence of pulmonary pleomorphic carcinoma was $0.90 \%$. This study was approved by the institutional review boards of each institute, and all patients provided written informed consent.

\subsection{CT and FDG-PET protocol}


Chest computed tomography (CT) was performed in all patients. The size, location, and internal density of the tumor, and the presence of a cavity were evaluated. Hilar and mediastinal lymph nodes were measured, and if the short-axis diameter of a lymph node was equal to or longer than $10 \mathrm{~mm}$, it was considered positive. Abdomen CT was also performed to detect metastases to abdominal organs such as liver, kidney, and adrenal gland.

Positron emission tomography with 18-fluorodeoxy-glucose (FDG-PET) has been used for evaluating tumors of the lung as well as lymph node and distant metastases. The Standardized Uptake Value (SUV), which was obtained by placing a region of interest over the lesion and dividing the value (in microcuries per cubic centimeter) by the injected dose (in microcuries) divided by the patient's body weight (in grams) [5], was measured. In this series, 12 patients underwent FDG-PET.

\subsection{Pathological diagnosis}

Pleomorphic carcinoma was defined as non-small cell carcinoma containing at least $10 \%$ sarcomatoid components. Pathologists evaluated specimens that were obtained by surgery, autopsy and TBB. In this study, when a TBB specimen contained both carcinomatous and sarcomatoid components, and the non-small cell lung cancer component was clearly distinct from the spindle/giant cell carcinoma, the tumor was diagnosed as pleomorphic carcinoma.

\subsection{Evaluation of response to chemotherapy or radiotherapy}


Response evaluations for chemotherapy and/or radiotherapy were assessed using RECIST (Response Evaluation Criteria in Solid Tumors) guidelines [6]. Overall survival was defined as the time from the first day of treatment until death from any cause.

\subsection{Statistical analysis}

In this paper, statistical values are given as mean \pm standard deviation. Continuous variables were compared using the Student t test or Mann-Whitney test, as appropriate. All survival curves for time-to-event variables were created using the Kaplan-Meier method [7].

\section{Results}

\subsection{Patient characteristics}

Patient characteristics are summarized in Table 1. The male:female ratio was 19:3. The age at diagnosis was $68.3 \pm 10.1$ years (range 51-94). Symptoms were seen in 16 patients (72.7\%): common symptoms were hemoptysis (seven cases) and cough (six cases). Fever, pain, dyspnea and body weight loss were also seen. Six patients (27.3\%) had no symptoms but were referred to hospital with lung nodules detected on chest X-rays. Eighteen patients (81.8\%) were current or ex-smokers, and the remaining four (18.2\%) 
had never smoked. The smoking history was striking: the mean number of pack-years of the 22 patients was $46.4 \pm 36.3$, with a maximum value of 160 pack-years (patient 5); the mean value of the 18 smokers was $56.7 \pm 31.8$ pack-years.

Biochemical examination revealed that in 14 of the 22 cases (63.6\%), serum carcinoembryonic antigen (CEA) was high, at $18.1 \pm 41.2 \mathrm{ng} / \mathrm{ml}$ (normal 1.0 6.5). In 10 of the 18 cases examined (55.6\%), cytokeratin fragment 19 (CYFRA 21-1) was also increased (4.0 $\pm 4.3 \mathrm{mg} / \mathrm{ml}$; range 0.0 - 14.1; normal $0.0-2.0)$. No other tumor markers examined, including carbohydrate antigen 19-9 (CA19-9), Sialyl Lewis (x) (SLX), squamous-cell carcinoma antigen (SCC-Ag), pro-gastrin-releasing peptide (Pro-GRP), and neuron-specific enolase (NSE), were specific and useful for diagnosis of pleomorphic carcinoma of the lung.

\subsection{Radiological findings}

Chest CT was performed in all 22 cases and revealed fairly large tumors: $45.3 \pm 21.9 \mathrm{~mm}$ in diameter (range $14-110$ ). All tumors were observed as mass or nodule, and ground glass opacity pattern or consolidations were not seen in our study. One case (patient 17) had a cavity inside the tumor (tumor diameter $50 \mathrm{~mm})$. Twenty-one tumors (95.5\%) were located in the peripheral field of the lung, while one was central (patient 20, non-small cell carcinoma + spindle cell). In 15 cases (68.2\%), the primary tumor was located in the upper lobe (right: 12 cases; left: 3 cases), and the remaining seven tumors (31.8\%) were located in the middle or lower lobe. Four cases exhibited internal low 
densities and heterogeneous pattern (patients 1, 14, 15, and 19). At the time of diagnosis, chest wall invasions were found in two patients (patients 6 and 17) and clinical T4 disease (malignant pleural and/or pericardial effusion, pleural dissemination, mediastinal invasion, and metastases to the same lobe) in four patients (patients 14, 16, 19, and 22). Clinical nodal involvements on CT findings were observed in 13 of the 22 patients ( 7 of 15 patients who underwent surgical resection).

Twelve patients underwent FDG-PET, and the SUV of the primary lesions tended to be high $(9.44 \pm 4.98$; range $3.00-16.6)$. SUVs of T1 $(\leq 3 \mathrm{~cm})$ and T2 $(>3 \mathrm{~cm})$ disease were $7.16 \pm 4.59$ and $9.89 \pm 5.16$, respectively. Most of the metastatic lymph nodes and distant metastases also exhibited high SUV.

\subsection{Diagnosis and pathological findings}

Fifteen cases of pulmonary pleomorphic carcinoma were diagnosed by surgical resection (Table 1; patients 1 - 15). Representative histological finding of the resected tumor is shown in Fig. $1 \mathrm{~A}$ (patient 4 , squamous cell carcinoma with spindle cell). Eleven of the 15 tumors were diagnosed as primary lung cancer by sputum and/or transbronchial brushing cytology, or TBB before surgery. Another four cases (Table 1; patients $16-19$ ) were confirmed by autopsy to have pulmonary pleomorphic carcinoma. In the other three patients (Table 1; patients 20 - 22), sarcomatoid elements were successfully detected with TBB (Fig. 1B, patient 21, large cell carcinoma with giant cell), and pleomorphic carcinoma was diagnosed without surgical resection or autopsy. 
Sputum cytology was performed in 15 patients. In two patients (13.3\%), carcinoma cells but not sarcomatoid elements were detected: one case (patient 10) was diagnosed as squamous cell carcinoma and the other (patient 4) as adenosquamous cell carcinoma.

Transbronchial brushing cytology and/or TBB was performed in 21 patients, confirming 17 cases of lung cancer. In 7 of the 17 cases, sarcomatoid elements were pathologically obtained in specimens. Three (patients 1, 9, and 11) of these patients received surgical resection, and one (patient 18) underwent autopsy. The other three (patients 20 - 22) patients were diagnosed with pleomorphic carcinoma by the TBB results alone without surgery or autopsy.

We also investigated differences in clinical features according to pathological subtypes. Large cell carcinoma combined with giant cell (six cases, 27.3\%) and adenocarcinoma combined with spindle cell (four cases, 18.2\%) were predominantly observed. We did not find any statistical differences in clinical or radiological characteristics according to pathological subtypes.

\subsection{Clinical course and prognosis for patients having surgical treatment}

Clinical stages at the point of diagnosis were stage IA in four cases (18.2\%), stage IB in three cases (13.6\%), stage IIB in four cases (18.2\%), stage IIIA in three cases (13.6\%), stage IIIB in three cases (13.6\%), and stage IV in five

cases $(22.7 \%)$ (Table 1$)$. Of the 15 patients with surgical resection (patients 1 15), pathological stage (p-stage) was upgraded compared with clinical stage (c-stage) in four cases (26.7\%). Pathological stages were stage IA in three 
cases (20\%), stage IB in three cases (20\%), stage IIB in four cases $(26.7 \%)$, stage IIIA in two cases (13.3\%), stage IIIB in two cases (13.3\%), and stage IV in one case (6.7\%) (Table 1). Fig. 2A shows overall Kaplan-Meier survival curve in all of the enrolled 22 patients. Median survival time (MST) was 213 days.

Of the 15 patients who underwent surgery, six patients, including five with pNO disease, relapsed after surgery. Four of them had recurrence by distant metastases (two to the brain, one to lung, and one to bone). Four of these patients died from relapsed cancer, while two patients (patients 9 and 15) are still alive (one of them is receiving gefitinib). The other nine patients have not developed any recurrence at the time of analysis, including two patients who died of non-cancer-related disease (pneumonia and heart disease).

Four patients (patients $1,6,10$, and 14) received adjuvant chemotherapy post-surgery. Patient 6 exhibited pT3NOMO (stage IIB) with chest wall invasion and received chemo-radiation therapy (cisplatin plus docetaxel and 65 Gy radiation in total). This patient was still alive without recurrence 441 days after surgery. Other three patients received chemotherapy alone. Patient 1 received UFT (Tegafur/Uracil) and was alive without recurrence 573 days after surgery. Patient 10 had adjuvant chemotherapy with vindesine, UFT, and OK432. This patient had recurrence 78 days after surgery and died after 126 days. Patient 14 received carboplatin and gemcitabine and was alive 98 days after surgery.

Patient 9 and 15 received chemotherapy for a recurrent tumor after surgery (Table 2). Patient 9 received cisplatin plus docetaxel as first-line therapy, and the response was stable disease (SD). It is noteworthy that the case (a 
70-year-old non-smoker woman) exhibited partial response to gefitinib as second-line therapy. The pathological subtype of this case was adenocarcinoma combined with spindle cell. Interestingly, the tumor harbored epidermal growth factor receptor (EGFR) mutations (L858R).

Fig. 2B shows overall Kaplan-Meier survival curve in the 15 patients who underwent surgical resection. Nine patients were still alive at the time of analysis, and MST was not determined.

3.5. Clinical course and prognosis for patients who did not undergo surgery but received chemotherapy or radiotherapy

Four (patients 16, 17, 19, and 21) of the seven cases who did not undergo surgical resection (patients $16-22$ ) and one (patient 15) of patients who received surgery had distant metastases at the time of diagnosis (stage IV). The metastasis sites were bone (two cases), lung, adrenal gland, skin, and lymph node (one case each). Three patients (patients 18, 20, and 22) did not have surgical treatment because they had c-stage IIIB disease (patients 18 and 22) or advanced age (patient 20; 94 years old). During their clinical course, other metastases to brain, liver and the small intestine appeared. One patient (patient 18) died of perforation of the small intestine because of metastasis. Autopsy (patients 16 - 19) revealed small metastases to heart, kidney, thyroid gland and distant lymph nodes that could not be proven by CT or MRI before death. Fig. 2B shows Kaplan-Meier overall survival curve for the seven patients who did not undergo surgery; MST was 118 days. 
Three patients received chemotherapy against advanced tumor without surgical resection (patients 17, 18, and 19). As first-line therapy, the patients received carboplatin plus paclitaxel (patients 17 and 18) and carboplatin plus docetaxel (patient 19). Two received gemcitabine plus vinorelbine as second-line treatment (patients 17 and 18). Only patient 17 received gefitinib as third-line and TS-1 as fourth-line treatments. Response was very poor, as shown in Table 2 (there were two NE (Not Evaluated) cases because of difficulties in performing follow-up examinations after onset of acute drug-induced pneumonitis). Even with first-line chemotherapy, no partial response was observed with the cytotoxic agents. The MST of these three patients (who did not undergo surgical treatment) was 213 days.

A total of six patients received irradiation for primary tumor causing back pain (patient 22), for brain metastasis (patient 8), for bone metastasis (patients 9 and 21), and as adjuvant therapy after surgical resection for pT3 tumors (patients 6 and 7).

Two of twenty-two patients received best supportive care because of advanced age and poor Eastern Cooperative Oncology Group Performance Status (ECOG PS) (patients 16 and 20).

\section{Discussion}

Lung tumors have been reclassified by the World Health Organization. In 1999, a group named "carcinoma with pleomorphic, sarcomatoid, or sarcomatous elements" was defined. In 2004, this group, which contained 
pleomorphic carcinoma, was renamed "Sarcomatoid carcinoma" [1]. Travis WD et al. had previously reported pleomorphic carcinoma of the lung to be very rare (approximately $0.1-0.4 \%$ of all lung malignancies) [2-4]. Since its pathological definition became widely recognized, pleomorphic carcinoma has been diagnosed more frequently: in our study, its frequency was $0.90 \%(22 / 2447$ cases). In another recent study, it was found to be 1.6\% (45/2743) of resected non-small cell lung cancer (NSCLC) [8]. As in previous reports [8-12], most cases in our study were male with a history of smoking; they exhibited symptoms such as hemoptysis and cough.

Pleomorphic carcinoma of the lung was often found as a large mass, more than $4-5 \mathrm{~cm}$ in diameter. In our study, the tumors tended to be located in the periphery of the upper lobes. Frequently observed hemoptysis might be characteristic of pulmonary pleomorphic carcinoma in spite of its peripheral location. We discovered sarcomatoid elements in seven cases by transbronchial brushing cytology and/or biopsy; however, there were no cases diagnosed only with cytological examination. Even when the component is included in the specimen, definite diagnosis of pleomorphic carcinoma should be avoided because it is difficult to differentiate the spindle component from active fibroblasts, as well as a giant cell component from multinucleated histiocytes. Kim et al. reported that $86 \%$ of pulmonary pleomorphic carcinomas with an adenocarcinoma component and $100 \%$ of those with a large cell carcinoma component were located in the periphery, while $100 \%$ of the pleomorphic carcinomas with a squamous cell carcinoma component were located in the central region [10]. They also demonstrated that the CT features of the tumor 
appeared to be dictated by its epithelial components. However, in our study, only one tumor was centrally located, the subtype of which was non-small cell carcinoma combined with spindle cell. All three cases of pulmonary pleomorphic carcinomas with a squamous cell carcinoma component were located in the periphery. In addition, CT features such as a cavity and heterogeneous density in the tumor that reflected necrosis or hemorrhage were not related to tumor subtypes. Neither patient characteristics (age, sex, smoking history, and symptoms), FDG-PET findings, response to chemotherapy, nor prognosis differed significantly between pathological subtypes. Mochizuki et al. also described that there were no significant differences in the overall survival between groups divided by predominant epithelial component [12].

FDG-PET was useful in examining patients with pleomorphic carcinoma of the lung, because it showed higher SUV in primary lesions even if the size of the tumor was small (minimum size was $15 \mathrm{~mm}$ in the 12 examined cases). SUVs of T1 $\leqslant 3 \mathrm{~cm})$ and T2 $-4(>3 \mathrm{~cm})$ disease were $7.16 \pm 4.59$ and $9.89 \pm 5.16$, respectively. Tournoy et al. has reported mean SUVs of T1 and T2-4 NSCLC as 3.71 and 5.20 [13]. There was a statistically significant difference in SUV of T2-4 diseases between NSCLC and pleomorphic carcinoma $(P<0.01)$, whereas there was no significant difference in SUV of T1 diseases $(P=0.48)$. Although there were only two T1 tumors in our study, our FDG-PET result suggests that the SUV of pleomorphic carcinoma might be higher than that of common non-small cell lung cancer, which might be helpful in diagnosis.

Biochemical examination revealed serum CEA to be elevated in $63.6 \%$ of patients (14 cases). This marker is known to be specific for other tumors 
including adenocarcinoma and is affected by external factors such as smoking. SCC, CYFRA, SLX, Pro-GRP, and NSE were considered to be non-specific markers for pulmonary pleomorphic carcinoma.

Distant metastasis was frequently observed in patients with pleomorphic carcinoma of the lung. The major sites of distant metastases were the same as those for other malignant epithelial lung tumors, i.e., bone, brain, lung, liver and adrenal gland, but the progression of this tumor is particularly rapid, and distant metastases seem to strongly influence prognosis. Autopsies revealed that pleomorphic carcinomas of the lung tended to expand into various lesions: minor sites of metastases such as thyroid grand, peritoneum and lymph nodes of abdomen could not be found by clinical examination before death. In particular, metastases to small intestine were revealed by autopsy in two patients, one of whose death (patient 18) was a result of intestine perforation.

Chest wall invasions and mediastinal invasions were commonly observed in the 15 patients who underwent surgical resection, as postoperative pathological findings rather than preoperative prediction. Because of pT3 disease, three cases were given adjuvant therapy (irradiation (patient 7) or chemotherapy (patient 1) or chemo-radiation therapy (patient 6)). Recurrence after surgery was frequently observed, and it should be noted that five cases with pNO disease also relapsed. While Raveglia et al. reported that nodal involvement was a determinant prognostic variable of pleomorphic carcinoma of the lung [9], Yuki et al. commented that even patients with pNO disease frequently experienced vascular invasion (57.1\%) [8]. We have to keep in mind that pleomorphic carcinoma of the lung has the potential to recur even if the primary 
lesion is resected at an early stage. In our study, the 15 patients who underwent surgery better prognoses, with nine patients surviving, compared with a MST of 118 days for patients who had not received surgery. One reason might be that some patients properly received chemotherapy, irradiation, or combination as adjuvant therapy post-surgery or when the relapsed tumor was found; indeed four patients receiving the treatments are still alive.

Chemotherapy was administered to nine patients, four of whom received adjuvant chemotherapy after surgery, while the other five had recurrence after surgery or advanced cases without surgery; however, neither platinum-based nor non-platinum-based chemotherapy was effective. Bae et al. also commented that advanced pulmonary pleomorphic carcinoma showed poor response to chemotherapy regimens [11]. Of note is the fact that we experienced one partial response to gefitinib despite this being second-line treatment (patient 9). The tumor in this case was diagnosed as pleomorphic carcinoma by surgical resection, and EGFR mutation (L858R) was detected. The presence of EGFR mutation is related to a patient's background and pathological subtypes of the lung cancer: it influences response to gefitinib [14]. This Japanese patient was female with no history of smoking, and the subtype of the tumor was adenocarcinoma combined with spindle cell. She had relapsed 19 months after surgery. First, she received platinum-based chemotherapy, which caused a SD response, and then gefitinib as second-line treatment resulted in survival for 890 days after surgery. It was not clear whether gefitinib was effective for only the adenocarcinoma component and not the spindle cell component since we could not obtain a tumor tissue sample after treatment. 
However, the good response to second-line gefitinib highlighted the importance of molecular targeted therapy for this entity.

\section{Conclusion}

In conclusion, we retrospectively analyzed 22 cases of pulmonary pleomorphic carcinoma in a total of 2447 cases of primary lung cancer. Pulmonary pleomorphic carcinoma had strong malignant potential with frequent distant metastases. We demonstrated that surgical treatment and appropriate follow-up therapy including the use of a molecular targeting drug (e.g., gefitinib for patients with adenocarcinoma as carcinomatous component) might improve outcomes. Some other important findings regarding the characteristics and clinical course of the tumor have been described in this report, and further investigations will be needed to elucidate more definitive clinical features and to establish appropriate methodological strategies for pleomorphic carcinoma of the lung.

\section{Acknowledgements}

We are grateful to Dr Koichi Yamazaki, former associate professor of the First Department of Medicine, Hokkaido University School of Medicine, for his outstanding support. We also thank Dr Yoshihiro Matsuno (Hokkaido University Hospital), Dr Katsushige Yamashiro (Hokkaido Cancer Center), Dr Kazuo Watanabe (Fukushima Medical University Hospital), Dr Yuichiro Fukasawa (KKR 
Sapporo Medical Center), and Dr Kenzo Okamoto (Hokkaido Chuo Rosai Hospital), for valuable assistance in pathological diagnosis and review. 


\section{References}

[1] Beasley MB, Brambilla E, Travis WD. The 2004 World Health Organization classification of lung tumors. Semin Roentgenol. 2005;40: 90-97.

[2] Brambilla E, Travis WD, Colby TV, Corrin B, Shimosato Y. The new World Health Organization classification of lung tumours. Eur Respir J. 2001;18: 1059-1068.

[3] Travis WD, Travis LB, Devesa SS. Lung cancer. Cancer. 1995;75: 191-202.

[4] Travis WD. Pathology of lung cancer. Clin Chest Med. 2002;23: 65-81.

[5] Rohren EM, Turkington TG, Coleman RE. Clinical applications of PET in oncology. Radiology. 2004;231: 305-332.

[6] Therasse P, Arbuck SG, Eisenhauer EA, Wanders J, Kaplan RS, Rubinstein L, Verweij J, Van Glabbeke M, van Oosterom AT, Christian MC, Gwyther SG. New guidelines to evaluate the response to treatment in solid tumors. J Natl Cancer Inst. 2000;92: 205-216.

[7] Kaplan EL, Meier P. Nonparametric estimation from incomplete observations. J Am Stat Assoc. 1958;53: 457-481.

[8] Yuki T, Sakuma T, Ohbayashi C, Yoshimura M, Tsubota N, Okita Y, Okada M. Pleomorphic carcinoma of the lung: a surgical outcome. J Thorac Cardiovasc Surg. 2007;134: 399-404.

[9] Raveglia F, Mezzetti M, Panigalli T, Furia S, Giuliani L, Conforti S, Meda S. Personal experience in surgical management of pulmonary pleomorphic carcinoma. Ann Thorac Surg. 2004;78: 1742-1747.

[10] Kim TS, Han J, Lee KS, Jeong YJ, Kwak SH, Byun HS, Chung MJ, Kim H, Kwon OJ. CT findings of surgically resected pleomorphic carcinoma of the lung 
in 30 patients. Am J Roentgenol. 2005;185: 120-125.

[11] Bae HM, Min HS, Lee SH, Kim DW, Chung DH, Lee JS, Kim YW, Heo DS. Palliative chemotherapy for pulmonary pleomorphic carcinoma. Lung Cancer. 2007;58: 112-115.

[12] Mochizuki T, Ishii G, Nagai K, Yoshida J, Nishimura M, Mizuno T, Yokose T, Suzuki K, Ochiai A. Pleomorphic carcinoma of the lung: clinicopathologic characteristics of 70 cases. Am J Surg Pathol. 2008;32: 1727-35.

[13] Tournoy KG, Maddens S, Gosselin R, Van Maele G, van Meerbeeck JP, Kelles A. Integrated FDG-PET/CT does not make invasive staging of the intrathoracic lymph nodes in non-small cell lung cancer redundant: a prospective study. Thorax. 2007;62: 696-701.

[14] Lynch TJ, Bell DW, Sordella R, Gurubhagavatula S, Okimoto RA, Brannigan BW, Harris PL, Haserlat SM, Supko JG, Haluska FG, Louis DN, Christiani DC, Settleman J, Haber DA. Activating mutations in the epidermal growth factor receptor underlying responsiveness of non-small-cell lung cancer to gefitinib. $\mathrm{N}$ Engl J Med. 2004;350: 2129-2139. 


\section{FIGURE LEGENDS}

Figure 1. Histological findings of pleomorphic carcinoma. (A) A spindle cell carcinoma component seen in a surgically resected tumor (Patient 4, hematoxylin and eosin, $\times 100)$. (B) A giant cell carcinoma component in a tumor tissue obtained by transbronchial biopsy (Patient 21, hematoxylin and eosin, $\times 100)$.

Figure 2. (A) Kaplan-Meier overall survival of all patients and (B) overall survival of patients treated with surgery (15 patients) or without surgery (7 patients). 


\section{(A)}



\section{(B)}

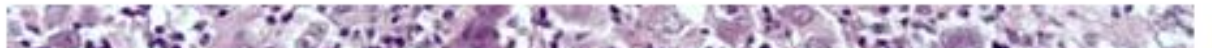

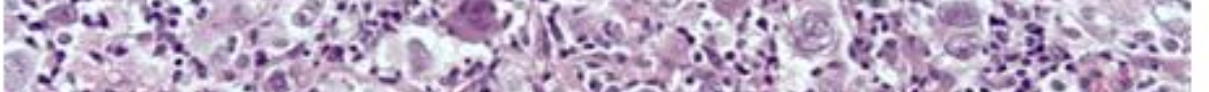



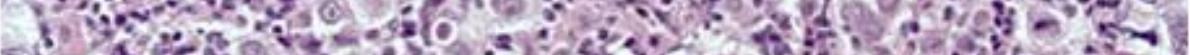

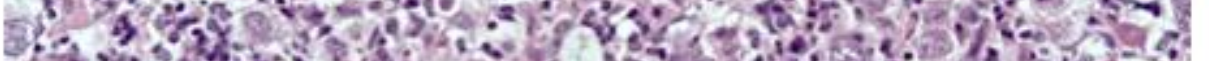

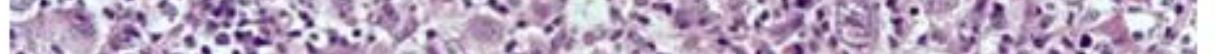


Prots

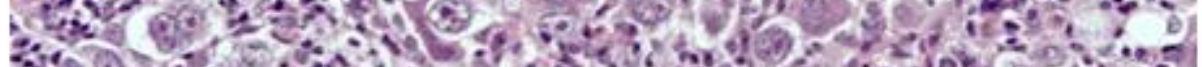

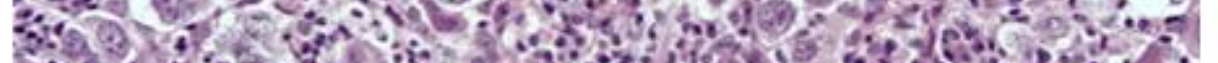

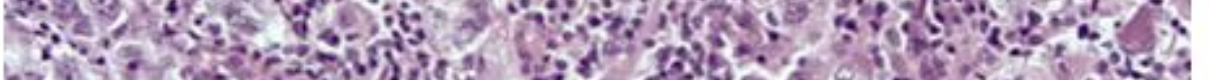



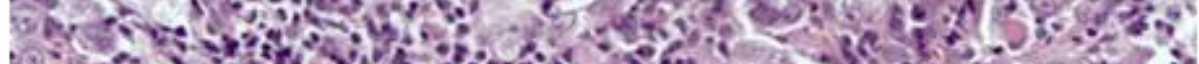

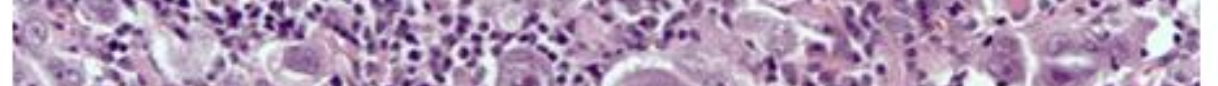

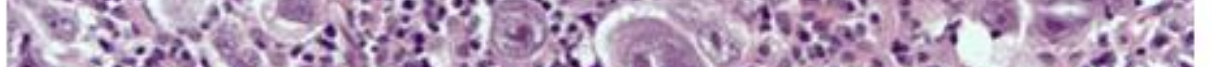

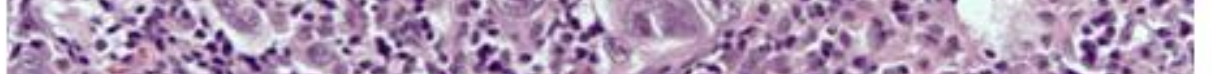
of

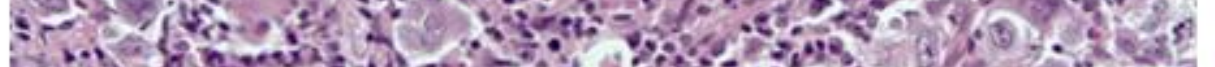

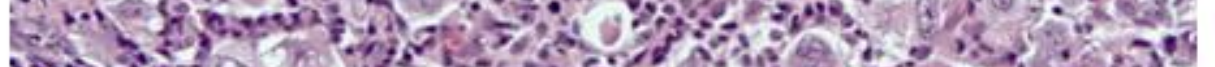

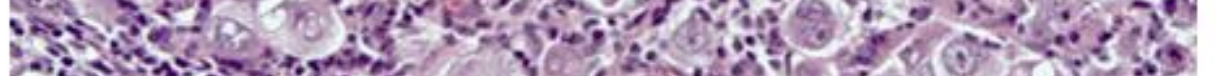

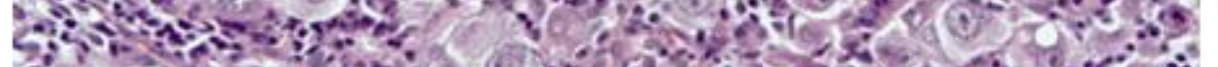

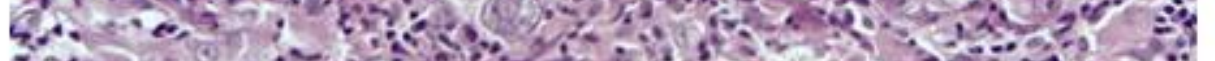

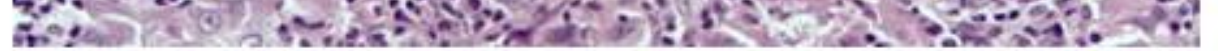




\section{(A)}

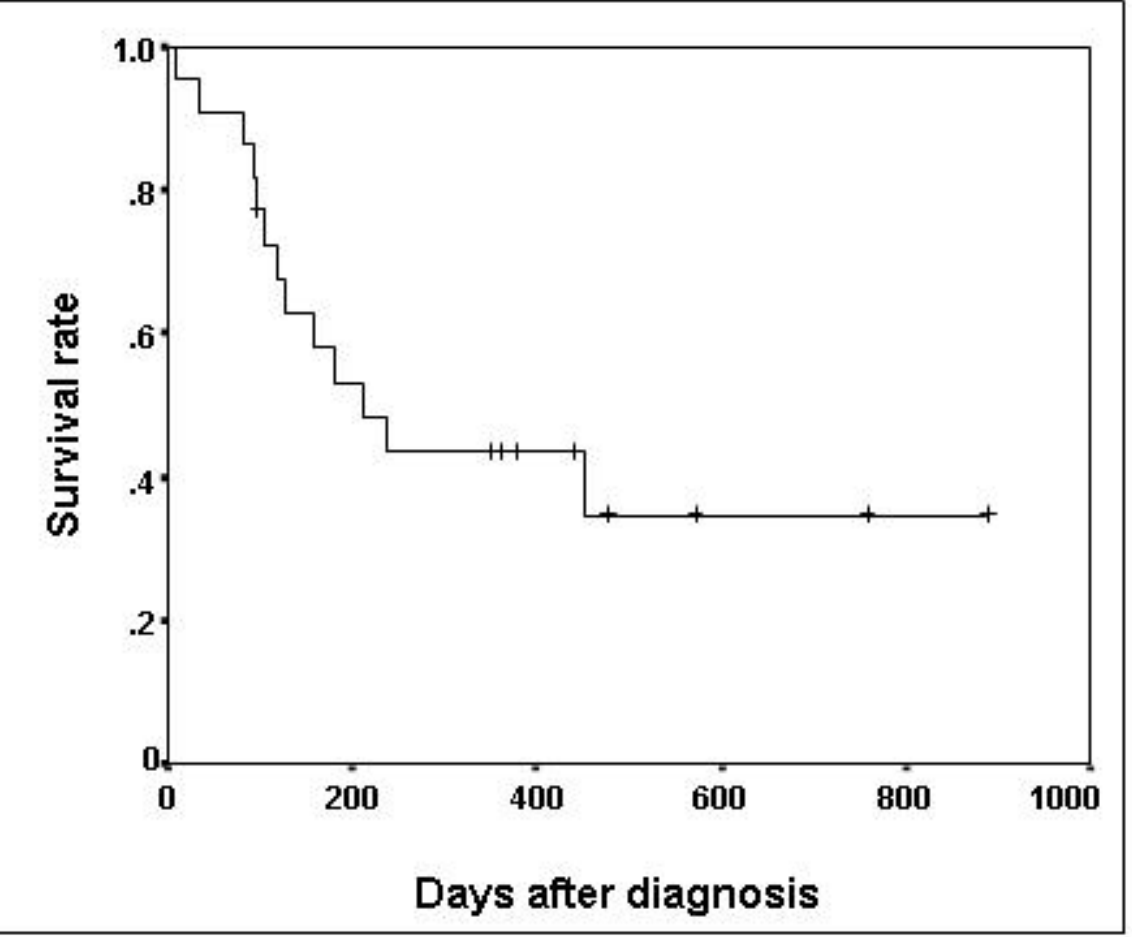

(B)




TABLE 1.

Clinical and pathological findings of 22 patients with pleomorphic carcinoma of the lung.

\begin{tabular}{|c|c|c|c|c|c|c|c|c|c|}
\hline \multirow{2}{*}{$\begin{array}{c}\text { Patient } \\
\text { No. }\end{array}$} & \multirow{2}{*}{ Sex } & \multirow{2}{*}{$\begin{array}{c}\text { Age } \\
\text { (years) }\end{array}$} & \multirow{2}{*}{$\begin{array}{c}\text { Smoking } \\
\text { history } \\
\text { (pack-years) }\end{array}$} & \multirow[t]{2}{*}{ Symptoms } & \multirow{2}{*}{$\begin{array}{l}\text { CEA } \\
(\mathrm{ng} / \mathrm{ml})\end{array}$} & \multicolumn{2}{|c|}{ Pathological subtypes } & \multirow[t]{2}{*}{ c-Stage } & \multirow[t]{2}{*}{ p-Stage } \\
\hline & & & & & & Carcinomatous component & Sarcomatoid component & & \\
\hline \multicolumn{10}{|c|}{ Surgical resection } \\
\hline 1 & M & 79 & 75 & $\begin{array}{l}\text { hemoptysis } \\
\text { body weight loss }\end{array}$ & 7.7 & Large cell carcinoma & Giant cell & cT2N1M0 IIB & pT3N2MO IIIA \\
\hline 2 & M & 67 & 42 & none & 12.4 & Adenocarcinoma & Giant cell & cT1NOMO IA & pT1NOMO IA \\
\hline 3 & M & 70 & 37.5 & fever & 8.8 & Large cell carcinoma & Giant cell & cT2N1M0 IIB & pT2NOMO IB \\
\hline 4 & M & 77 & 35.6 & hemoptysis & 8.1 & Squamous cell carcinoma & Spindle cell & cT2N2M0 IIIA & pT4N2M0 IIIB \\
\hline 5 & M & 77 & 160 & none & 9.8 & Large cell carcinoma & Spindle cell & cT2N1M0 IIB & pT2N1M0 IIB \\
\hline 6 & M & 59 & 51.3 & none & 6.6 & Adenosquamous cell carcinoma & Spindle cell & cT3NOMO IIB & рT3NOMO IIB \\
\hline 7 & M & 64 & 66 & $\begin{array}{l}\text { hemoptysis } \\
\text { cough }\end{array}$ & 2.3 & Adenocarcinoma & Spindle cell & cT2NOMO IB & pT3NOMO IIB \\
\hline 8 & M & 64 & 67.5 & hemoptysis & 7.5 & Adenocarcinoma & Spindle cell & cT2NOMO IB & pT2NOMO IB \\
\hline 9 & $\mathrm{~F}$ & 70 & 0 & cough & 12.8 & Adenocarcinoma & Spindle cell & cT1NOMO IA & PT1NOMO IA \\
\hline 10 & M & 73 & 31.8 & hemoptysis & 8.3 & Squamous cell carcinoma & Spindle cell + Giant cell & cT2N2M0 IIIA & pT2N2MO IIIA \\
\hline 11 & M & 71 & 56 & dyspnea & 3.4 & Adenocarcinoma & Giant cell & cT2N2M0 IIIA & pT2N1M0 IIB \\
\hline 12 & M & 74 & 50 & hemoptysis & 2.8 & Large cell carcinoma & Giant cell & cT2NOMO IB & pT2NOMO IB \\
\hline 13 & M & 68 & 55 & none & 1.3 & Large cell carcinoma & Giant cell & cT1NOMO IA & PT1NOMO IA \\
\hline 14 & M & 51 & 33.8 & $\begin{array}{l}\text { dyspnea } \\
\text { cough }\end{array}$ & 0.7 & Non-small cell carcinoma & Giant cell & CT4NOMO IIIB & pT4NOMO IIIB \\
\hline 15 & M & 54 & 82.5 & none & 2.7 & Adenocarcinoma & Giant cell & cT2N2M1 IV & pT4NOM1 IV \\
\hline \multicolumn{10}{|l|}{ Autopsy } \\
\hline 16 & M & 73 & 75 & $\begin{array}{l}\text { dyspnea } \\
\text { back pain }\end{array}$ & 197 & Large cell carcinoma & Spindle cell + Giant cell & cT4N3M1 IV & \\
\hline 17 & M & 52 & 30 & $\begin{array}{l}\text { hemoptysis } \\
\text { cough }\end{array}$ & 37.2 & Squamous cell carcinoma & Spindle cell + Giant cell & cT3N2M1 IV & \\
\hline 18 & M & 57 & 60 & $\begin{array}{l}\text { cough, sputum } \\
\text { fever }\end{array}$ & 15.4 & Large cell carcinoma & Giant cell & cT2N3M0 IIIB & \\
\hline 19 & $\mathrm{~F}$ & 63 & 0 & cough, fever & 4.4 & Adenocarcinoma & Giant cell & cT4N1M1 IV & \\
\hline \multicolumn{10}{|c|}{ TBB (transbronchial biopsy) } \\
\hline 20 & M & 94 & 0 & none & 8.1 & Non-small cell carcinoma & Spindle cell & cT1NOMO IA & \\
\hline 21 & $\mathrm{~F}$ & 70 & 0 & none & 38.4 & Large cell carcinoma & Giant cell & cT2N3M1 IV & \\
\hline 22 & M & 76 & 12.5 & back pain & 2.9 & Adenocarcinoma & Spindle cell & cT4N2M0 IIIB & \\
\hline
\end{tabular}


TABLE 2.

Summary of chemotherapy for 5 patients except for adjuvant therapy.

Patient No. Situation Stage $\quad$ Regimens (Best Response)

\begin{tabular}{|c|c|c|}
\hline 9 & recurrence after surgery pT1NOMO IA & CDDP/DOC $(\mathrm{SD}) \rightarrow$ Gef $(\mathrm{PR})$ \\
\hline 15 & recurrence after surgery pT4NOM1 IV & CBDCA/PTX (SD) \\
\hline 17 & no indication of surgery cT3N2M1 IV & CBDCA/PTX $(\mathrm{SD}) \rightarrow$ GEM/VNR $(\mathrm{PD}) \rightarrow \operatorname{Gef}\left(\mathrm{NE}^{*}\right) \rightarrow \mathrm{S}-1(\mathrm{PD})$ \\
\hline 18 & no indication of surgery cT2N3MO IIIB & CBDCA/PTX $(\mathrm{SD}) \rightarrow$ GEM/VNR $\left(\mathrm{NE}^{*}\right)$ \\
\hline 19 & no indication of surgery cT4N1M1 IV & CBDCA/DOC (SD) \\
\hline
\end{tabular}

*occurred drug-induced interstitial pneumonitis

CDDP: cisplatin, DOC: docetaxel, Gef: gefitinib, CBDCA: carboplatin, PTX: paclitaxel, GEM: gemcitabine, VNR: vinorelbine, S-1: TS-1, PR: partial response, SD: stable disease, PD: progressive disease, NE: not evaluated 